\title{
RIEMANN'S METHOD IN THE THEORY OF SPECIAL FUNCTIONS
}

\author{
J. SCHWARTZ 1
}

To E. L. Post

The theory of special functions is known as a "painful" subject. My talk today might as well be entitled, "special functions without pain." The painful nature of the subject springs from this: that at first sight, it seems to consist of an enormous number of uncorrelated individual cases, the particular significance, connection, or depth of any specific formula being hard to judge-an enormous mass of chaotic detail, the worst sort of material with which to deal. What is needed, then, is some way of ordering and correlating the material, which should, in order of importance, accomplish the following ends.

(1) To make evident in a general intuitive way what sorts of formulae are likely to hold "on standard grounds" and, conversely, which formulae depend on really special or on deeper properties of a given special function.

(2) To give in a mechanical way the exact form of as wide a class of formulae as possible.

(3) To furnish exact proofs of these formulae.

In attempting to develop such a scheme, it is clear that the question of notation will play a role of importance. In contrast to the conventionally meaningless ad hoc notations for the various kinds of special functions, one wants to develop a "natural" notation, which will bring out as much of the structure of a given function as possible. What I would like to do today is to indicate how one such system can be developed, to indicate the derivation of a few formulae on the basis of this system, and to describe in a general way the range of applicability of the methods involved.

The complete system would consist of three parts:

(a) the function theoretic method (Riemann's method);

(b) the Laplace transform method;

(c) the method of partial differential equations.

An address delivered before the New York Meeting of the Society, April 20, 1956, by invitation of the Committee to Select Hour Speakers for Eastern Sectional Meetings; received by the editors May 24, 1956.

1 The research contained in this paper was supported by a grant from the Office of Naval Research. Reproduction in whole or in part for any purpose of the United States Government is permitted. 
Today I shall discuss only the first of these, since the latter two are more standard. However, I should remark that

(a) covers transformation, recursion, differentiation, multiplication, and a number of expansion formulae, and provides a general framework into which (b) and (c) fit.

(b) covers most integral formulae, if used in a sufficiently systematic way. For an excellent introduction to the application of the Laplace transform method, the recent book of van der Pol and Bremmer may be consulted.

(c) covers a class of addition and expansion formulae. For a most interesting treatment of this method, the papers of P. Henrici listed in the bibliography appended to the present paper should be consulted.

Together, (a), (b), and (c), if used systematically, can cover over $80 \%$ at least of the body of formulae constituting the standard corpus of "special function theory," leaving a residue of $20 \%$ or so which count from the point of view of (a), (b), and (c) as special formulae.

The basic idea of the method (a) is taken from Riemann's famous paper of 1857 on the hypergeometric function, and is simply thisto characterize a differential equation with rational coefficients not in terms of the parameters entering into its coefficients, but in terms of its singularities, and in terms of parameters describing the nature of its solutions in the neighborhood of these singularities. We shall consequently consider an ordinary homogeneous linear differential equation of order $n$

$$
(L f)(z)=f^{(n)}(z)+r_{n-1}(z) f^{(n-1)}(z)+\cdots+r_{0}(z) f(z)=0,
$$

the coefficients $r_{j}$ being rational functions. A "special function" will be a solution of such an equation. This definition includes all the "special functions of mathematical physics"-hypergeometric, Whittaker, Bessel, Lame, Mathieu, ellipsoidal wave, etc., functions, but excludes abelian integrals (though not strictly), $\theta$-functions, the gamma function, etc.

The singularities of equation (1) are $z=\infty$ and the singularities of its coefficients. As is customary, we distinguish the singularities into regular and irregular singularities, and the irregular singularities into irregular singularities of various types. This may be done in the conventional way by examining the orders of the poles of the coefficients $r_{f}$ at the various singularities. More significantly for the present purpose, it may be done by considering the behavior of the solutions of (1) at a singularity $\zeta$. For simplicity, take $\zeta=0$. Then it is known that in general (1) possesses linearly independent solutions 


$$
\sigma_{1}, \cdots, \sigma_{n}
$$

which are asymptotically of the form

$$
\begin{aligned}
\sigma_{i}(z) \sim \exp \left(q_{i, k_{i}} z^{-k_{i} / \nu_{i}}+\cdots+q_{i, 1} z^{-1 / \nu_{i}}\right) z^{\alpha_{i}}(1 & +p_{i, 1} z^{1 / \nu_{i}} \\
& \left.+p_{i, 2} z^{2 / \nu_{i}}+\cdots\right),
\end{aligned}
$$

in the neighborhood of $z=0, \nu_{i}$ being a certain integer. The series here are not convergent but only asymptotic, and that only in certain angular sectors of the plane. Moreover, these series may in particular cases contain logarithmic terms also. However, for simplicity in the present presentation, I will ignore these possibilities.

As a convenient symbolic representation of the asymptotic series (2), let us write

$$
\left[\nu_{i} \mid q_{i, k_{i}}, \cdots, q_{i, 1}, \alpha_{i} ; p_{i, 1}, \cdots, p_{i, N_{i}}\right]
$$

to as many terms as is profitable, calling $N_{i}$ the number of times the symbol (3) is augmented. We normally assume that $q_{i, k_{i}}$ is not zero; that is, if $q_{i, l}=0$ for $l \geqq p+1$, we normally begin the symbol (3) with $\left[\nu_{i} \mid q_{i, p}, \cdots\right]$. If $\nu_{i}=1$, it will normally be omitted from the symbol (3). Finally, we agree to write $[\alpha]$ as $\alpha$. Then if the asymptotic forms of the solutions of equation (1), $L f=0$, at the singularity $\zeta=0$ are represented by the symbols (3), the type $\tau(\zeta)$ of the singularity $\zeta$ is given by the simple formula

$$
\tau(\zeta)=\text { smallest integer not less than } \max _{t=1 \ldots n}\left(k_{i} / \nu_{i}\right)+1
$$

If $\tau(\zeta)=1$, the singularity is regular; otherwise it is irregular.

The quantity $R$ defined by the sum $R=\sum \tau(\zeta)$ extended over all the finite and infinite singularities of the equation (1) may be called the Riemann number of equation (1). The Riemann number $R$ measures the total number of singularities of equation (1), counted according to type. If we count the total number $P=P(R, n)$ of parameters on which an equation of Riemann number $R$ and order $n$ depends, we easily find

$$
P(R, n)=(R-2) \frac{n(n+1)}{2}+n .
$$

The symbol (3) has been introduced as a description of the asymptotic form of a given solution of equation (1) at one of its singularities $\zeta$. The singularity $\zeta$ itself may now be described by the collection of symbols (3) describing the $n$ asymptotic forms of $n$ linearly independent solutions a $\zeta$, that is, by the collection of symbols 


$$
\begin{gathered}
\zeta \\
{\left[\nu_{1} \mid q_{1, k_{1}}, \cdots, \alpha_{1} ; p_{1,1}, \cdots, p_{1, N_{1}}\right],} \\
{\left[\nu_{2} \mid q_{2, k_{2}}, \cdots, \alpha_{2} ; p_{2,1}, \cdots, p_{2, N_{2}}\right],} \\
\cdots \cdots, \cdots \cdots, \\
{\left[\nu_{n} \mid q_{n, k_{n}}, \cdots, \alpha_{n} ; p_{n, 1}, \cdots, p_{n, N_{n}}\right] .}
\end{gathered}
$$

The quantities $\alpha_{1}, \cdots, \alpha_{n}$ are called the exponents of the singularity $\zeta$. If (6) describes a single singularity of equation (1), and if $\zeta_{1}, \cdots, \zeta_{p}$ is the collection of all singularities of equation (1), it is natural to describe equation (1) itself by the conglomerate symbol

$$
\begin{aligned}
& \zeta_{1} \quad \zeta_{2} \cdots \zeta_{p}
\end{aligned}
$$

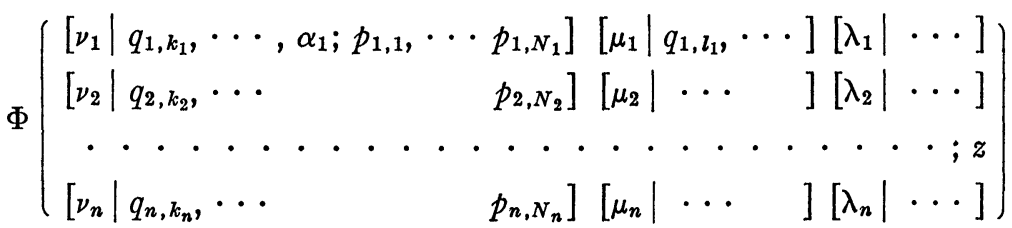

composed of the symbolic descriptions of all its singularities. The symbol (7) may be called the Riemann symbol of equation (1). I have followed Riemann in writing it as a symbolic function of $z$, which may, according to the requirements of convenience, denote either the equation (1) itself, or an arbitrary branch of the multi-valued function which is its solution. The parameters entering into the generalized Riemann symbol (7) are not all independent; the exponents are bound together by the generalized Fuchs relation, which may be stated in case equation (1) has regular singularities only in the form

$$
\sum \alpha_{i}=\frac{n(n-1)}{2}(R-2),
$$

the sum in (8) being extended over all singularities and all exponents. Relation (8) holds even in the presence of irregular singularities in suitable "nondegenerate" cases. In the presence of "degeneracies," formula (8) must be appropriately modified.

By augmenting the entries in the Riemann symbol (7) a sufficient number of times, we may ensure that the symbol (7) contains at least as many independent parameters as the number (5) of parameters entering into the coefficients of equation (1). In this case, it is reasonable to surmise that the symbol (7) determines the corresponding equation (1) uniquely. It is in fact easy to state general conditions under which the symbolic description (7) determines the corresponding equation uniquely. Without stating these conditions explicitly, 
I shall write only such symbols (7) as do determine a unique differential equation. To find the symbolic representation of a given equation (1), we have only to substitute trial solutions of the form (2) into equation (1), and to compare the coefficients of the lowest few powers of $z$ on the left and right of (1). This will yield the symbolic description of (1) after a small amount of algebraic computation.

Thus, for example, the hypergeometric equation

$$
\delta(\delta+\alpha-1) f(z)-z\left(\delta+\gamma_{1}\right)\left(\delta+\gamma_{2}\right) f(z)=0, \quad \delta=z d / d z,
$$

is associated with Riemann's symbol

$$
\Phi\left(\begin{array}{ccc}
0 & 1 & \infty \\
0 & 0 & \gamma_{1} ; z \\
1-\alpha & \alpha-\gamma_{1}-\gamma_{2} & \gamma_{2}
\end{array}\right) ;
$$

the confluent hypergeometric equation

$$
\delta(\delta+\alpha-1) f(z)-z(\delta+\gamma) f(z)=0, \quad \delta=z d / d z,
$$

with the symbol

$$
\Phi\left(\begin{array}{ccr}
0 & \infty \\
0 & \gamma \\
1-\alpha & {[1, \alpha-\gamma]}
\end{array}\right) .
$$

More generally, the generalized hypergeometric equation

$$
\begin{array}{r}
\delta\left(\delta+\alpha_{1}-1\right) \cdots\left(\delta+\alpha_{q}-1\right) f(z)-z\left(\delta+\gamma_{1}\right) \cdots\left(\delta+\gamma_{p}\right) f(z)=0 \\
\delta=z d / d z
\end{array}
$$

is associated if $p \leqq q$ with the symbol

$$
\Phi\left(\begin{array}{cc}
0 & \infty \\
0 & \gamma_{1} \\
1-\alpha_{1} & \dot{\gamma_{p}} \quad ; z \\
\dot{\cdot} & {\left[e \mid e \omega_{1}, f\right]} \\
\dot{1}-\dot{\alpha_{q}} & {\left[\dot{e} \mid \dot{e} \omega_{\epsilon}, \dot{f}\right]}
\end{array}\right),
$$

where $e=q+1-p ; \omega_{1}, \cdots, \omega_{e}$ are the $e$ th roots of unity, and $f=e^{-1}\left((q+p) / 2-\sum_{i=1}^{q} \alpha_{i}-\sum_{i=1}^{p} \gamma_{i}\right)$. In order to write a Riemann symbol characterizing the Fuchsian case $p=q+1$ of equation (9), it is convenient to introduce an auxiliary notational convention. It is 
known that if a group of several of the exponents at a regular singularity of equation (1) differ by an integer, the corresponding solutions may possibly contain logarithmic terms. If in a particular case such terms may apparently but do not in actuality appear, we indicate this by bracketing the corresponding entries in the associated Riemann symbol together on the left by a single curly bracket. In terms of this notational convention, the Fuchsian case $p=q+1$ of equation (9) is determined by the Riemann symbol

$$
\Phi\left(\begin{array}{ccc}
0 & 1 & \infty \\
0 & \\
1-\alpha_{1} & \vdots & \gamma_{1} \\
\vdots & \vdots & z \\
1-\alpha_{q} & \sum \alpha-\sum \gamma & \gamma_{p}
\end{array}\right) .
$$

As a final example, illustrating the employment of an augmented Riemann symbol, let me mention that the equation

$$
\left(1-z^{2}\right) f^{\prime \prime}(z)-2 z f^{\prime}(z)+\left(\lambda+4 \theta\left(1-z^{2}\right)-\mu^{2}\left(1-z^{2}\right)^{-1}\right) f(z)=0
$$

of spheroidal wave functions corresponds to the symbol

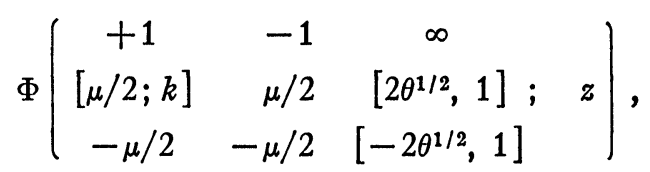

where

$$
k=k(\lambda, \theta, \mu)
$$

is an easily computed algebraic function. This symbol might also be written as

$$
\Phi\left(\begin{array}{ccc}
+1 & -1 & \infty \\
{[\mu / 2 ; k]} & {\left[\mu / 2 ; e^{-\pi i(1+\mu / 2)} k\right]} & {\left[2 \theta^{1 / 2}, 1\right] ; z} \\
-\mu / 2 & -\mu / 2 & {\left[-2 \theta^{1 / 2}, 1\right]}
\end{array}\right),
$$

indicating explicitly how the fact that the spheroidal wave equation is even is reflected in the corresponding symbol.

As a first example illustrating the use of the Riemann symbols (7) in the derivation of identities among special functions, let me note that since $e^{(-z)}=e^{-z}$, since $(-z)^{\beta}$ is a constant multiple of $z^{\beta}$, and since $e^{-x}$ is regular and nonvanishing in the finite plane, we have 


$$
\begin{aligned}
& \Phi\left(\begin{array}{ccc}
0 & \infty & \\
0 & \gamma & ; \\
1-\alpha & {[1, \alpha-\gamma]}
\end{array}\right)=\Phi\left(\begin{array}{cc}
0 & \infty \\
0 & \gamma \\
1-\alpha & {[-1, \alpha-\gamma]}
\end{array}\right) \\
& =e^{-z} \Phi\left(\begin{array}{cc}
0 & \infty \\
0 & {[1, \gamma] ; z} \\
1-\alpha & \alpha-\gamma
\end{array}\right)=e^{-z} \Phi\left(\begin{array}{cc}
0 & \infty \\
0 & \alpha-\gamma ; z \\
1-\alpha & {[1, \gamma]}
\end{array}\right) .
\end{aligned}
$$

Note that the Riemann symbol at the end of equation (10) is of the same general form as that which begins equation (10). If we write equation (10) in the more conventional notation of Pochammer for confluent hypergeometric functions, we see that we have established Kummer's transformation

$$
{ }_{1} F_{1}(\gamma ; \alpha ;-z)=e^{-z} F_{1}(\alpha-\gamma ; \alpha ; z) .
$$

It is easy to see, making use of the Riemann symbol for the generalized hypergeometric equation (9) in case $p \leqq q$, that this is the only formula of its sort which applies to the solutions of equation (9) in case $p \leqq q$. A more fanciful change of variable, which applies to the solutions of equation (9) if $p=3, q=2$, and which may be justified in the same way as (10), is

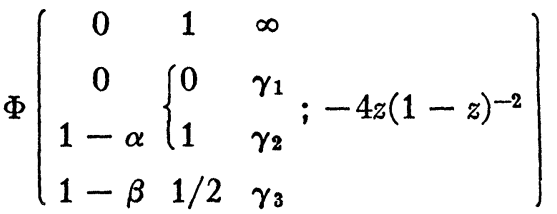

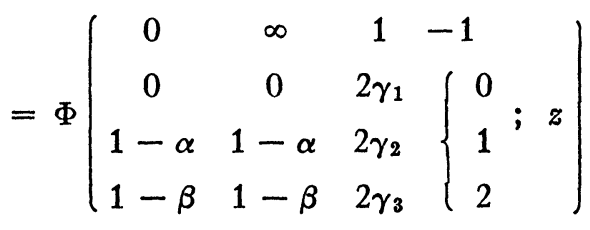

$$
\begin{aligned}
& =\Phi\left(\begin{array}{ccc}
0 & 1 & \infty \\
0 & 2 \gamma_{1} & 0 \\
1-\alpha & 2 \gamma_{2} & 1-\alpha \\
1-\beta & 2 \gamma_{3} & 1-\beta
\end{array}\right) ; z \\
& =(1-z)^{2 \gamma_{1}} \Phi\left(\begin{array}{ccc}
0 & 1 & \infty \\
0 & 0 & 2 \gamma_{1} \\
1-\alpha & 2\left(\gamma_{2}-\gamma_{1}\right) & 1-\alpha+2 \gamma_{1} \\
1-\beta & 2\left(\gamma_{3}-\gamma_{1}\right) & 1-\beta+2 \gamma_{1}
\end{array} ; z\right) \text {. }
\end{aligned}
$$


In case $\gamma_{2}=\gamma_{1}+1 / 2$, the last Riemann symbol in this equation evidently denotes a solution of (9) with $p=3, q=2$, and we have evidently derived Whipple's transformation

$$
\begin{aligned}
{ }_{3} F_{2}\left(\gamma_{1}, \gamma_{1}+\right. & \left.1 / 2, \gamma_{3} ; \alpha, \beta ;-4 z(1-z)^{-2}\right) \\
& =(1-z)^{2 \gamma_{1} F_{2}}\left(2 \gamma_{1}, 2 \gamma_{1}+1-\alpha, 2 \gamma_{1}+1-\beta ; \alpha, \beta ; z\right) .
\end{aligned}
$$

Let us now consider the process of multiplying two functions. For this purpose, first note that the differential equation with singlevalued coefficients of lowest order satisfied by a given multi-valued function is the smallest number of its branches on which all its branches are linearly dependent. Suppose then that we take two multi-valued functions $\sigma$ and $\rho$, each satisfying a second order differential equation with single-valued coefficients. Then there exist two branches $\sigma_{1}, \sigma_{2}$ of $\sigma$ on which all the continuations of $\sigma$ are linearly dependent, and two branches $\rho_{1}, \rho_{2}$ of $\rho$ on which all the branches of $\rho$ are linearly dependent. It is then apparent that all the branches of the product $\sigma \rho$ are dependent on the four branches

$$
\sigma_{1}, \rho_{1}, \quad \sigma_{2} \rho_{2}, \quad \sigma_{1} \rho_{2}, \quad \sigma_{2} \rho_{1} .
$$

This observation justifies the following calculation.

$$
\begin{aligned}
& \left.\Phi\left(\begin{array}{cc}
0 & \infty \\
0 & \gamma \\
1-\alpha & {[1, \alpha-\gamma]}
\end{array}\right) \quad z\right) \Phi\left(\begin{array}{cc}
0 & \infty \\
0 & \gamma \\
1-\alpha & {[1, \alpha-\gamma]}
\end{array}\right) \\
& =\Phi\left(\begin{array}{cc}
0 & \infty \\
{[0 ; 0]} & 2 \gamma \\
2-2 \alpha & 2(\alpha-\gamma) ; \quad z \\
1-\alpha & {[1, \alpha]} \\
2-\alpha & {[-1, \alpha]}
\end{array}\right) \\
& =\Phi\left(\begin{array}{cc}
0 & \infty \\
0 & \gamma \\
1-\alpha & \alpha-\gamma \quad ; z^{2} / 4 \\
1 / 2-\alpha / 2 & {[2 \mid 2, \alpha / 2]} \\
1-\alpha / 2 & {[2 \mid-2, \alpha / 2]}
\end{array}\right) .
\end{aligned}
$$

In more conventional notation, this is the following formula of Ramanujan

(11) ${ }_{1} F_{1}(\alpha ; \gamma ; z){ }_{1} F_{1}(\alpha ; \gamma ;-z)-{ }_{2} F_{3}\left(\gamma, \alpha-\gamma ; \alpha, \alpha / 2, \alpha / 2+1 / 2 ; z^{2} / 4\right)$. 
An amusing example of the sort of formula of this type sometimes to be met is the following formula of Bailey.

$$
\begin{aligned}
{ }_{0} F_{2}\left(\rho_{1}, \rho_{2} ; z\right){ }_{0} F_{2}\left(\rho_{1}, \rho_{2} ;-z\right)={ }_{3} F_{8} \\
=\left[\frac{1}{3}\left(\rho_{1}+\rho_{2}-1\right), \frac{1}{3}\left(\rho_{1}+\rho_{2}\right), \frac{1}{3}\left(\rho_{1}+\rho_{2}+1\right) ;\right. \\
-\rho_{1}, \rho_{2}, \frac{1}{2} \rho_{1}, \frac{1}{2} \rho_{2}, \frac{1}{2}\left(\rho_{1}+1\right), \frac{1}{2}\left(\rho_{2}+1\right), \\
\left.\frac{1}{2}\left(\rho_{1}+\rho_{2}-1\right), \frac{1}{2}\left(\rho_{1}+\rho_{2}\right) ;\left(\frac{3}{4}\right){ }^{3} z^{2}\right] .
\end{aligned}
$$

The derivation of (12) by Riemann's method is just as trivial as the derivation of (11).

As a final example, we calculate the Laplace transform of the square of a Bessel function, so as to illustrate the way in which the function theoretic method links up with the method of the Laplace transform. Rather than giving the explicit statement of the various general rules governing the Laplace transform of a Riemann symbol, we will display the various steps of the calculation, which will make the principles involved reasonably clear. If $\& f$ denotes the Laplace transform of $f$, we have

$$
\begin{aligned}
& \int_{0}^{\infty} e^{-s t}\left(J_{\nu}(t)\right)^{2} d t=\mathscr{L}\left(\Phi\left(\begin{array}{cc}
0 & \infty \\
\nu & {[i, 1 / 2] ;} \\
-\nu & {[-i, 1 / 2]}
\end{array}\right)^{2}\right)(s) \\
& =\mathscr{S}\left(\Phi\left(\begin{array}{cc}
0 & \infty \\
{[0 ; 0]} & 1 \\
2 \nu & {[2 i, 1]} \\
-2 \nu & {[-2 i, 2]}
\end{array}\right)\right)(s) \\
& =\Phi\left(\begin{array}{cccc}
2 i & -2 i & 0 & \infty \\
0 & 0 & {[0 ; 0]} & 1-2 \nu ; s \\
0 & 0 & 0 & 1+2 \nu
\end{array}\right) \\
& =\Phi\left(\begin{array}{rrc}
0 & -4 & \infty \\
0 & 0 & 1 / 2-\nu ; s^{2} \\
0 & 0 & 1 / 2+\nu
\end{array}\right) \\
& =\Phi\left(\begin{array}{rrcc}
-1 & +1 & \infty & \\
0 & 0 & 1 / 2-\nu ; & \frac{s^{2}+2}{2} \\
0 & 0 & 1 / 2+\nu
\end{array}\right) \text {. }
\end{aligned}
$$


Here we have used most notably the fact that the Laplace transform of a function satisfying a differential equation with (even) rational coefficients satisfies a differential equation with even rational coefficients. By noting the asymptotic form of the Laplace integral in (13) as $s \rightarrow \infty$ we may determine the particular branch of

$$
\Phi\left(\begin{array}{rrcc}
-1 & +1 & \infty \\
0 & 0 & 1 / 2-\nu ; \frac{s^{2}+2}{2} \\
0 & 0 & 1 / 2+\nu
\end{array}\right)
$$

represented by this integral. In this way we find that the integral in (13) represents the Legendre function of the second kind, and that (13) may be written in more conventional notation as

$$
\int_{0}^{\infty} e^{-s t}\left(J_{\nu}(t)\right)^{2} d t=\frac{1}{\pi} Q_{v-1 / 2}\left(\frac{s^{2}+2}{2}\right) .
$$

This is a special case of a formula of Hankel.

\section{BIBLIOGRAPHY}

A. Erdélyi et al.

1. Higher transcendental functions, vols. 1-3, New York, 1953.

P. Henrici

1. On generating functions of the Jacobi polynomials, Pacific J. Math. vol. 5, supplement 2 (1955) pp. 923-931.

2. Addition theorems for general Legendre and Gegenbauer functions, Journal of Rational Mechanics and Analysis, vol. 4, no. 6 (1955) pp. 983-1017.

3. Zur Funktionentheorie der Wellergleichung, Comment. Math. Helv. vol. 27 (1953) pp. 235-293.

B. van der Pol and $\mathrm{H}$. Bremmer

1. Operational calculus based on the two-sided Laplace integral, Cambridge University Press, 1955.

C. Truesdell

1. An essay toward a unified theory of special functions, Princeton, 1948.

YaLe UNIVERSity 